\title{
Abdominal imaging findings on computed tomography in patients acutely infected with SARS-CoV-2: what are the findings?
}

\author{
Michio Taya ${ }^{1} \cdot$ Viktoriya Paroder $^{2} \cdot$ Gil Redelman-Sidi $^{3} \cdot$ Natalie Gangai $^{2} \cdot$ Jennifer S. Golia Pernicka ${ }^{2}$. \\ Marc J. Gollub ${ }^{2} \cdot$ Sidra Javed-Tayyab ${ }^{2} \cdot$ Iva Petkovska $^{2} \cdot$ David D. B. Bates $^{2}$ (D)
}

Received: 5 August 2021 / Accepted: 6 September 2021 / Published online: 3 October 2021

(c) American Society of Emergency Radiology 2021

\begin{abstract}
Objectives To investigate what findings are new on contrast-enhanced abdominopelvic CT in patients infected with SARS-CoV-2.

Methods Contrast-enhanced CT of the abdomen and pelvis of patients with COVID-19 at a tertiary oncologic center acquired over a 2-month period were reviewed independently by two readers and scored for new imaging abnormalities compared with a prior scan. CT scans were included if the study was performed between -3 and 45 days from the time of COVID-19 diagnosis. Clinical information was gathered from the medical records.

Results A total of 63 patients ( 34 male, 29 female; mean age 60.6 years, range $24.4-85.0$ years) were included in this retrospective cross-sectional study. Aside from new ground glass opacities seen at the lung bases $(29 / 63,46.0 \%)$, the most common findings were new thickening of the stomach, small bowel or colon or fluid-filled colon $(14 / 63,22.2 \%)$, new small volume ascites $(7 / 63,14.3 \%)$, gallbladder distention in those without prior cholecystectomy $(3 / 43,7.0 \%)$, and single cases each of acute pancreatitis $(1 / 63,1.6 \%)$ as well as new portal vein thrombosis $(1 / 63,1.6 \%)$.

Conclusion Aside from lung base ground glass opacities, the most common new imaging abnormality on abdominopelvic CT in patients with COVID-19 finding in our cohort was abnormalities of the gastrointestinal tract, followed by small volume ascites, gallbladder distention, and isolated cases of pancreatitis and portal vein thrombosis. These findings overlap with those previously reported that did not have a prior scan for comparison, and provide supportive evidence that some of these findings may be related to SARS-CoV-2 infection.
\end{abstract}

Keywords COVID-19 $\cdot$ SARS-CoV-2 $\cdot$ Computed tomography $\cdot$ Coronavirus $\cdot$ Enterocolitis

$\begin{array}{ll}\text { Abbreviations } & \\ \text { COVID-19 } & \begin{array}{l}\text { Coronavirus disease 2019 } \\ \text { Severe acute respiratory syndrome corona- } \\ \text { virus 2 }\end{array} \\ \text { CT } & \begin{array}{l}\text { Computed tomography } \\ \text { SMV }\end{array} \\ \text { Superior mesenteric vein }\end{array}$

David D. B. Bates

batesd@mskcc.org

1 Department of Radiology, Weill Cornell Medical College, New York, NY, USA

2 Department of Radiology, Memorial Sloan Kettering Cancer Center, 1275 York Ave, New York, NY 10065, USA

3 Department of Infectious Disease, Memorial Sloan Kettering Cancer Center, New York, NY, USA

\section{Introduction}

In late 2019, a novel coronavirus emerged that spread quickly and resulted in a global pandemic. At the time of this writing, severe acute respiratory syndrome coronavirus 2 (SARS-CoV-2) and the infection it causes, coronavirus disease 2019 (COVID-19), have wrought havoc worldwide. The World Health Organization currently reports 192,284,207 confirmed cases with $4,136,518$ confirmed deaths worldwide [1].

Although COVID-19 primarily manifests as a respiratory pathogen causing pneumonia and respiratory failure [2-4], much remains unknown about this relatively new pathogen and its involvement of other organ systems. Abnormalities of the enteric tract have been reported in previously published radiologic series [5, 6], and SARS-CoV-2 RNA has been isolated in the duodenal wall and feces of some patients [7]. Furthermore, wastewater has been identified as a means to 
monitor levels of COVID-19 in communities [8, 9], implying that it may be shed from the gastrointestinal tract. This is not entirely unexpected, as SARS-CoV-2 is known to enter host cells via the angiotensin-converting enzyme 2 (ACE2) cell receptor, which is expressed in the esophagus, ileum, and colon in addition to the lung [10].

Apart from gastrointestinal findings, radiological abnormalities of other abdominal organs have been reported in patients with COVID-19, including of the biliary tract, abdominal vasculature, and pancreas [5, 6, 11, 12]. However, currently published studies do not compare imaging of patients with COVID-19 to their pre-COVID-19 imaging studies to establish whether observed abnormalities are new since infection with the virus. A previous study has explored the unique elements of COVID-19 in oncology patients [13], but to our knowledge, no paper has focused on imaging specifically in cancer patients infected with SARSCoV-2. This study investigates a series of oncologic patients with COVID-19 who underwent contrast-enhanced CT of the abdomen and pelvis, all of whom also had a prior CT available for comparison. In contrast, many previous studies either do not compare abdominal imaging findings to prior studies or rely exclusively on pre-existing radiology reports without review of the images.

\section{Methods}

Institutional review board approval was obtained for this retrospective cross-sectional study; informed consent was waived.

\section{Cohort selection}

A data query was placed through the hospital medical record system for all patients diagnosed with COVID-19 in March or April 2020, who had also undergone contrast-enhanced computed tomography imaging through the abdomen and pelvis at our hospital. Inclusion criteria were adult patients ( $>18$ years old) with computed tomography scans of the abdomen and pelvis performed with intravenous contrast that were performed in the time period between 3 days prior to 6 weeks after COVID-19 diagnosis. Subjects were also required to have a prior $\mathrm{CT}$ of the abdomen and pelvis available for comparison. As the study was conducted at a tertiary cancer center, all patients were oncology patients with prior CT scans of the abdomen and pelvis available for comparison.

\section{Image interpretation, scoring, and analysis}

Two radiologists, a fellowship-trained body radiologist (VP, 7 years of experience) and a radiology resident (MT), independently reviewed contrast-enhanced CT scans for 63 patients diagnosed with COVID-19. Using the software program REDCap, they recorded whether the following findings were present, and whether they were new from the prior comparison CT scan: ground glass opacities at the lung bases, gastric wall thickening, small bowel wall thickening greater than $3 \mathrm{~mm}$, colonic wall thickening greater than $3 \mathrm{~mm}$, fluid-filled colon, gallbladder distension greater than $10 \mathrm{~cm}$ in length or $4 \mathrm{~cm}$ in diameter, gallbladder thickening greater than $3 \mathrm{~mm}$, ascites, pancreatitis, superior mesenteric vein thrombosis, interstitial nephritis, hepatomegaly, or splenomegaly. Additional record was made whether gastric wall thickening was focal or diffuse, small bowel wall thickening involved a short $(<10 \mathrm{~cm})$ or long segment $(>10 \mathrm{~cm})$, whether it involved proximal, middle, or distal small bowel, what segment of the colon was thickened (ascending, transverse, descending/sigmoid, or rectal), whether ascites was small/moderate/large volume, and whether there was pancreatic necrosis. Spleen and hepatic craniocaudal heights were measured at the mid clavicular line. Disagreements were settled by a third reader, an abdominal radiologist with expertise in oncologic imaging (DB, 4 years of experience). A summary of the items scored is presented in Table 1. Descriptive statistics were performed (Table 1).

\section{Results}

Initially, 73 patients were identified as potential subjects. Ultimately, 10 were excluded: 7 patients had CT scans without IV contrast, 2 had no prior CT for comparison, and 1 had a positive COVID-19 test out of the date range. The final cohort included 63 patients, 34 male and 29 female, with a mean age of 60.6 years (range 24.4-85.0 years). The contrast-enhanced CT scans were performed on average 21 days after the diagnosis of COVID-19 (range -3 to 44 days) (Fig. 1).

The top 3 most common cancer diagnoses in our cohort were, in descending order, colon cancer $(8 / 63,12.7 \%)$, pancreatic cancer $(7 / 63,11.1 \%)$, and bladder cancer $(6 / 63$, $9.5 \%)$. A summary of patients' oncologic diagnoses and patient demographics are listed in Table 2.

Most patients [46/63 (73.0\%)] were scanned in the outpatient setting, while 9/63 (14.3\%) were inpatients, 7/63 $(11.1 \%)$ were scanned from the hospital urgent care, and $1 / 63(1.6 \%)$ was scanned while admitted to the intensive care unit. The mean time between the prior CT scan used for comparison and the index CT used for scoring was 69 days (range 0-432).

The most common finding on abdominopelvic CT was new ground glass opacities at the lung bases seen in 29/63 $(46.0 \%)$ of all cases in the cohort. The interobserver agreement for ground glass opacities at the lung bases was $84.1 \%$. 
Table 1 Scoresheet for readers to assess contrast-enhanced CT of the abdomen and pelvis
Did the patient have presence of ground glass opacities at the lung bases?

If yes, was the presence of ground glass opacities present on previous CT?

Did the patient have gastric thickening?

If yes, was gastric thickening present on prior CT?

If yes, was the gastric thickening local or diffuse?

Was there small bowel thickening $>0.3 \mathrm{~cm}$ ?

If yes, was small bowel thickening present on previous CT?

If yes, where?

If yes, how large?

Was there colonic thickening?

If yes, was colonic thickening present on the previous CT?

If yes, where was the colonic thickening? (choice = ascending)

If yes, where was the colonic thickening? $($ choice $=$ transverse $)$

If yes, where was the colonic thickening? $($ choice $=$ distal $)$

If yes, where was the colonic thickening? $($ choice $=$ rectum $)$

Did the patient have ascites?

If yes, was ascites present on the previous CT?

If yes, what was the volume?

Did the patient have pancreatitis?

If yes, was there pancreatitis present on previous CT?

If yes, was pancreatic necrosis present?

Is there gallbladder distention $>10-\mathrm{cm}$ length or $4-\mathrm{cm}$ diameter?

If yes, was there gallbladder distention present on the previous CT?

Was there gallbladder wall thickening?

If yes, was there presence of gallbladder wall thickening on previous CT?

Did the patient have splenomegaly $>13 \mathrm{~cm}$ craniocaudal?

If yes, was there presence of splenomegaly on the previous CT?

If yes, what was the measurement 1 on prior CT?

If yes, measurement 1 on COVID CT

Did the patient have hepatomegaly $>16 \mathrm{~cm}$ craniocaudal?

If yes, was there hepatomegaly present on the previous CT?

If yes, craniocaudal measurement at prior $\mathrm{CT}$

If yes, craniocaudal measurement at COVID CT

Was there presence of SMV or portal vein thrombosis?

If yes, was there presence of SMV or portal vein thrombus on previous CT?

Was there presence of interstitial nephritis?

If yes, was there presence of interstitial nephritis on previous CT?

Did the patient have presence of fluid-filled colon?

If yes, was there presence of fluid-filled colon on previous CT?
The second most common finding was a new abnormality of the gastrointestinal tract, with $14 / 63$ (22.2\%) patients showing one or more of the following: new wall thickening of either the stomach, small bowel, or colon, or new fluid-filled colon. New colonic wall thickening was most common, seen in 9/63 (14.3\% of cases), followed by new small bowel wall thickening in $4 / 63$ cases $(6.3 \%)$ and new gastric wall thickening seen in $2 / 63$ (3.2\%) of cases. A total of $3 / 63$ patients $(4.8 \%)$ were found to have a fluidfilled colon on CT, new from the prior scan. A total of $6 / 14$ $(40.0 \%)$ cases showed isolated colonic wall thickening,
3/14 (21.4\%) showed isolated small bowel wall thickening, and $1 / 14(7.1 \%)$ each showed isolated gastric wall thickening, isolated fluid-filled colon, combined colonic and gastric wall thickening, combined colonic wall thickening with fluid-filled colon, and combined small bowel and colonic wall thickening with fluid-filled colon. The interobserver agreement for colonic, small bowel, and gastric wall thickening was $95.2 \%, 85.7 \%$, and $95.2 \%$, respectively. Interobserver agreement for the category of fluid-filled colon was $98.4 \%$ (Figs. 2, 3, 4, and 5). 
Fig. 1 Flow chart for patient selection in the final study cohort
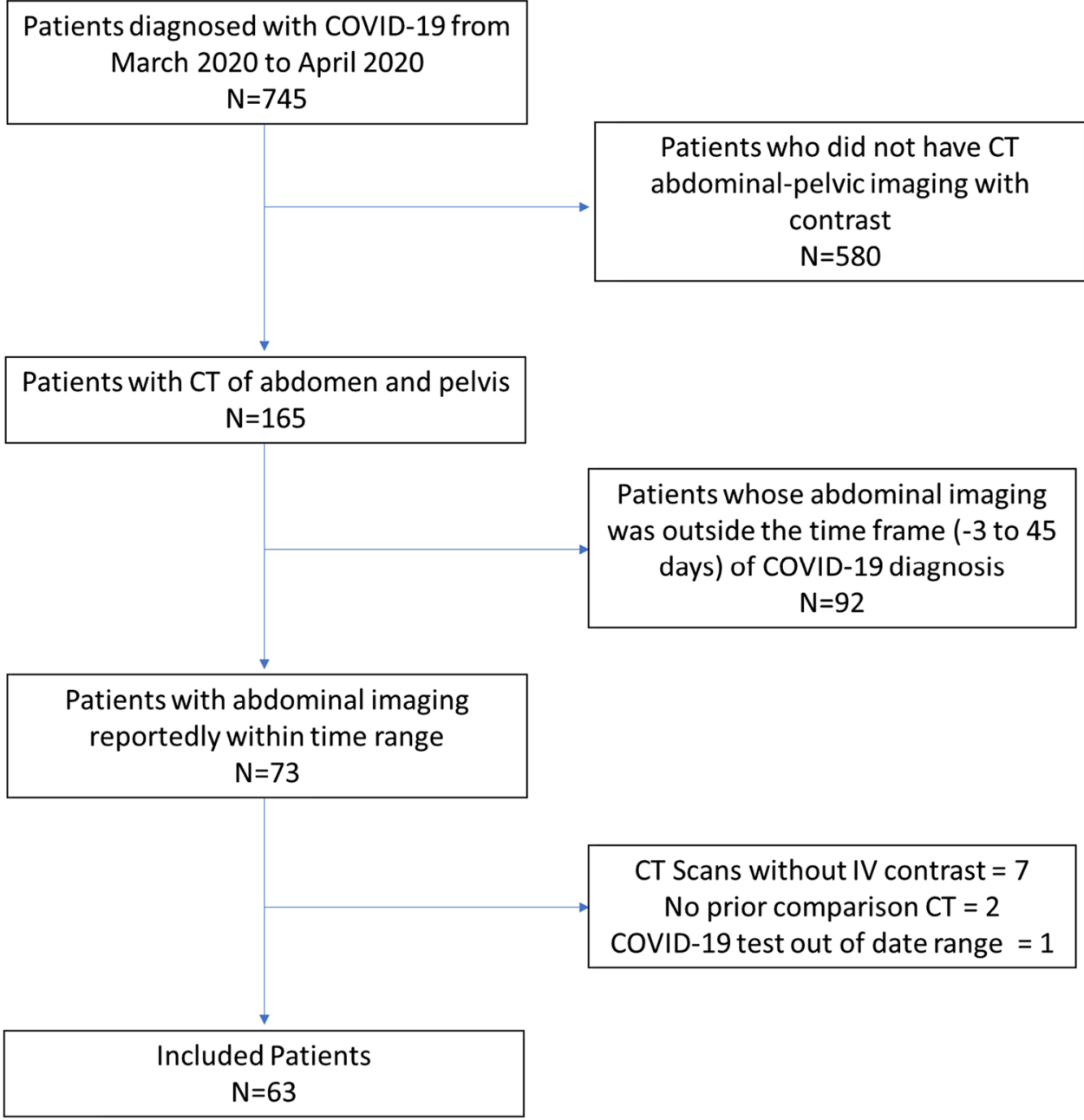

Among the 9 patients with new colonic wall thickening, the most commonly affected segment was the descending/ distal colon $(8 / 9,88.9 \%)$, followed by the transverse colon $(6 / 9,66.7 \%)$, the ascending colon $(5 / 9,55.6 \%)$, and lastly the rectum $(5 / 9,55.6 \%)$. Among the 4 patients where new small bowel wall thickening was seen, it was more commonly present in the proximal small bowel $(3 / 4,75.0 \%)$ and more often involved a long segment of bowel greater than $10 \mathrm{~cm}$ in length $(3 / 4,75 \%)$. Between the two patients with new gastric wall thickening, one case showed diffuse thickening and one was focal.

A total of $7 / 63(14.3 \%)$ of patients had new ascites. Among those with new ascites, it was considered small volume in $6 / 7(85.7 \%)$. The interobserver agreement for the presence of ascites was $88.9 \%$.

Twenty patients $(20 / 63,31.7 \%)$ had previously undergone cholecystectomy. Among the 43 patients who had not undergone cholecystectomy previously, new gallbladder distention was present in $3 / 43(7.0 \%)$ of cases. None of the patients showed gallbladder thickening. The interobserver agreement was $95.2 \%$ and $100.0 \%$ for gallbladder distention and gallbladder thickening, respectively.

There was a single case of new portal vein thrombosis $(1 / 63,1.6 \%)$ and no cases of SMV thrombosis. The interobserver agreement was $98.4 \%$.

There was a single case of new pancreatitis $(1 / 63,1.6 \%)$. There was no associated pancreatic necrosis. The interobserver agreement for pancreatitis was $96.8 \%$.

No patients had new splenomegaly or new hepatomegaly. A total of 11/63 (17.5\%) patients demonstrated pre-existing splenomegaly, with a mean craniocaudal splenic length of $14.6 \mathrm{~cm}$ that was unchanged from the prior scan. A total of $8 / 63(12.7 \%)$ patients demonstrated pre-existing hepatomegaly, with a mean craniocaudal length of $19.2 \mathrm{~cm}$, also unchanged from the prior scan. The interobserver agreement for splenomegaly was $82.5 \%$ and for hepatomegaly was $93.7 \%$.

None of the patients had CT evidence of interstitial nephritis. The interobserver agreement for interstitial nephritis was $100.0 \%$. 
Table 2 Summary of patient demographics and cancer types in the study cohort

\begin{tabular}{lll}
\hline Cancer type & & \\
\hline Colon & 8 & $12.7 \%$ \\
Pancreatic & 7 & $11.1 \%$ \\
Bladder & 6 & $9.5 \%$ \\
Cholangiocarcinoma & 4 & $6.3 \%$ \\
Lung cancer & 4 & $6.3 \%$ \\
Ovarian & 4 & $6.3 \%$ \\
Endometrial & 3 & $4.8 \%$ \\
Gastric & 3 & $4.8 \%$ \\
Prostate & 3 & $4.8 \%$ \\
Renal & 3 & $4.8 \%$ \\
Gallbladder & 2 & $3.2 \%$ \\
Rectal & 2 & $3.2 \%$ \\
Sarcoma & 2 & $3.2 \%$ \\
Appendiceal & 1 & $1.6 \%$ \\
Benign hepatic tumor & 1 & $1.6 \%$ \\
Breast & 1 & $1.6 \%$ \\
Cervical & 1 & $1.6 \%$ \\
GIST & 1 & $1.6 \%$ \\
Liposarcoma & 1 & $1.6 \%$ \\
Lymphoma & 1 & $1.6 \%$ \\
Neuroendocrine & 1 & $1.6 \%$ \\
Testicular & 1 & $1.6 \%$ \\
Urothelial & 1 & \\
Uterine & 1 & \\
Vulvar & 24.4 & \\
Demographics & 1 & \\
M & 1 & \\
F & & \\
Mean age (years) & & \\
Min & 1 & \\
Max & 1 & \\
\hline
\end{tabular}

Only 10/63 (15.9\%) patients reported at least one abdominal symptom such as abdominal pain $(7 / 63,11.1 \%)$, abdominal distention $(2 / 63,3.2 \%)$, diarrhea $(2 / 63,3.2 \%)$, or nausea/vomiting $(1 / 63,1.6 \%)$. Among patients with at least one abdominal symptom, one had new gastric wall thickening, one had new small bowel wall thickening, three had new colonic wall thickening, one had new presence of a fluidfilled colon, four had new small volume ascites, one had new pancreatitis, and one had new gallbladder distention without wall thickening.

\section{Discussion}

Among 63 patients with COVID-19, by far the most common finding on contrast-enhanced CT of the abdomen and pelvis was new ground glass opacities at the lung bases. Below the diaphragm, the most common findings were seen in the gastrointestinal tract (thickening of the stomach, small bowel, or colon, or fluid-filled colon), present in $22.2 \%$ of patients in our cohort. The next most common finding, the presence of new ascites as seen in $14.3 \%$ of our patients, was small volume in each case. Gallbladder distention without gallbladder thickening was seen in $9.3 \%$ of patients who has not previously undergone cholecystectomy. A single case each of new portal vein thrombosis and acute pancreatitis account for $1.6 \%$ of cases apiece in our study apiece.

The finding of abnormalities related to the stomach, small bowel, and colon may be related to patients' infection with the novel coronavirus. However, it is difficult to make a definitive link without a gold standard, such as pathologic correlation. Supporting the theory of viral involvement of the GI tract is the method by which COVID-19 enters cells. One of the key elements for cell entry for COVID-19 is the receptor for angiotensin-converting enzyme 2 (ACE-2),
Fig. 2 Chart summarizing the distribution of GI tract findings seen in patients with COVID-19 and new abnormalities of the stomach, small bowel, or colon on contrast-enhanced CT

\section{Distribution of GI tract findings when present in patients with COVID-19 on contrast-enhanced abdominal CT}

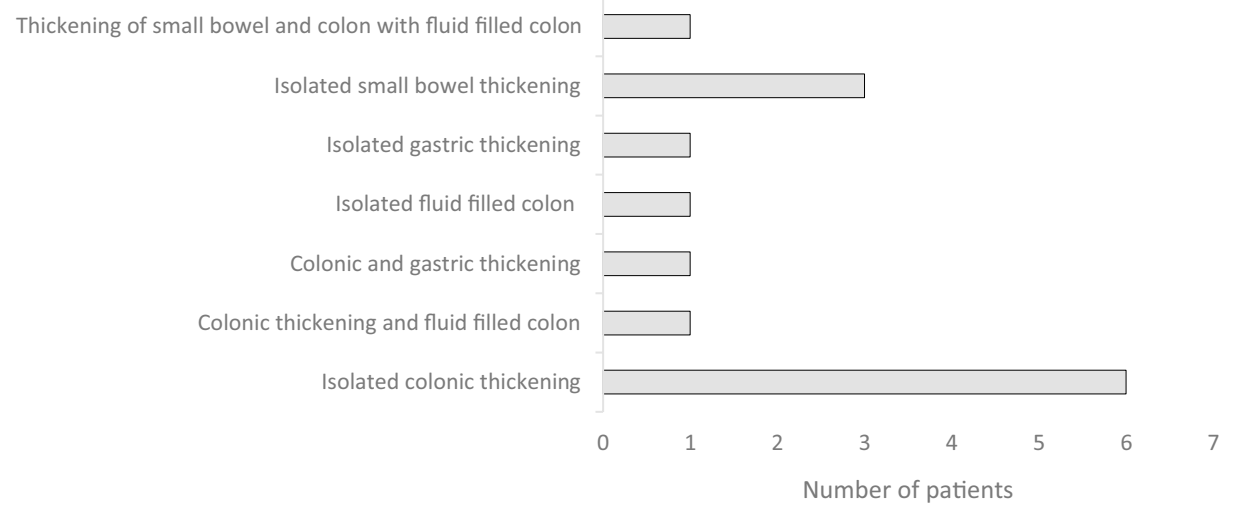




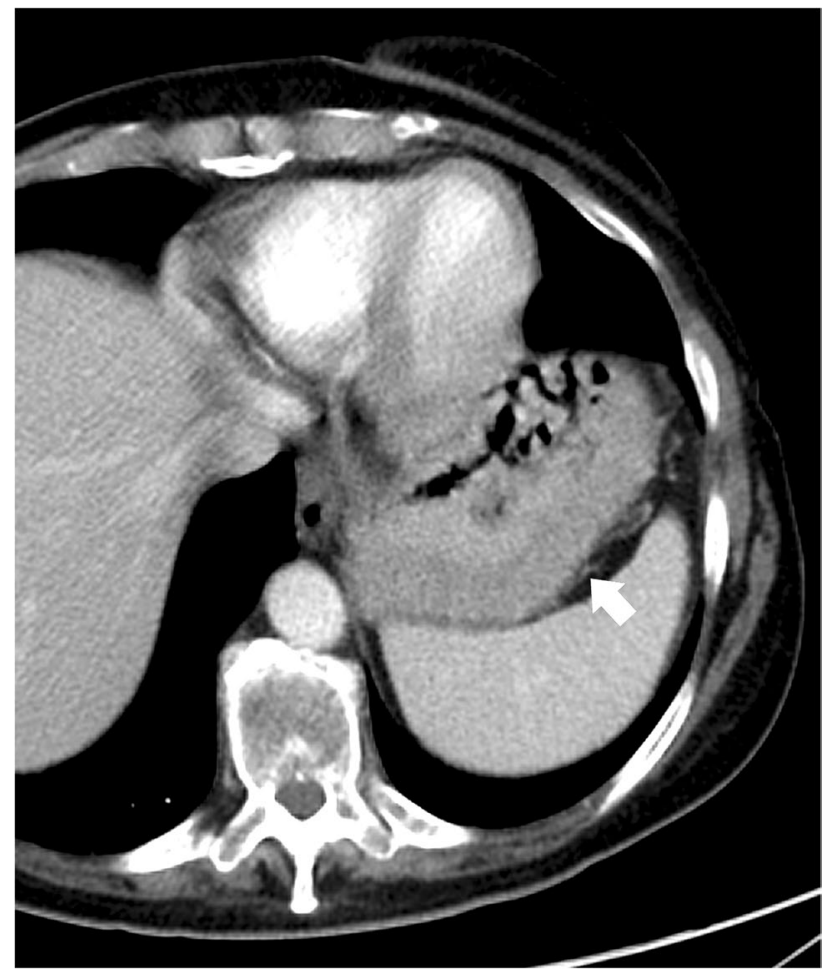

Fig. 3 A 72-year-old female with endometrial cancer and thickening of the gastric fundus (arrows) after diagnosis with COVID-19, which was new from the prior scan

which is present both in alveolar cells of the lung [14] and in the small bowel and colon $[10,15]$. The presence of small volume ascites in a small number of patients may be related to other factors, such as iatrogenic hydration or volume shifts related to acute illness, rather than being a direct result of viral infection. In $9.3 \%$ of patients with gallbladder distention, there was no associated gallbladder thickening or abnormality to suggest an inflammatory process, so this may be attributed to other benign causes such as the fasting status of the patients. The isolated case of pancreatitis could perhaps be related to COVID-19 infection, but without a pathologic reference standard, it may be equally likely attributable to other common causes of acute pancreatitis, including idiopathic pancreatitis. In the one case of new portal vein thrombosis, the patient also demonstrated progression of intrahepatic cholangiocarcinoma, which can be associated with portal vein thrombus, so caution is advised against drawing a conclusion as to a cause-effect relationship for this case.

Previously published studies provide context within which these results must be interpreted. By far, the most common finding on abdominal CT in patients with COVID19 is new ground glass opacities at the lung bases, seen in $46.0 \%$ of our cohort. This finding has also been reported in a number of other studies [16-22], as would be expected given that the virus is primarily manifested as a respiratory pathogen.

Taking the results of our study in context of existing literature, inferring what may be the clinical implications of this new data is the next step. The most common and widely reported finding, new ground glass opacities in the lung bases, is important for abdominal radiologists to keep in mind. Rarely, accurate identification of lung base findings on abdominal CT may be the first sign that a patient is infected with COVID-19 [20, 21], and the radiologist may be the one to suggest testing. More commonly, it is incidentally noted in patients already diagnosed with the virus [19-22].

The second most common finding in our cohort, new wall thickening of the stomach, small bowel, or colon, or fluid-filled colon, was seen in $22.2 \%$ and is perhaps the most interesting in terms of clinical implications. Patients infected with COVID-19 presenting with predominantly abdominal symptoms have been well documented in the literature [20, 22-31]. Although some of the reported cases with abdominal pain have no abnormality on CT [23], others have bowel abnormalities such as duodenojejunitis [24], venous and
Fig. 4 A 68-year-old male with colon cancer presents with new small bowel wall thickening. Axial images show loops of small bowel in the midabdomen with wall thickening greater than $3 \mathrm{~mm}$ (a, arrows), also shown in the coronal reformat (b, arrow). There is also moderate volume ascites
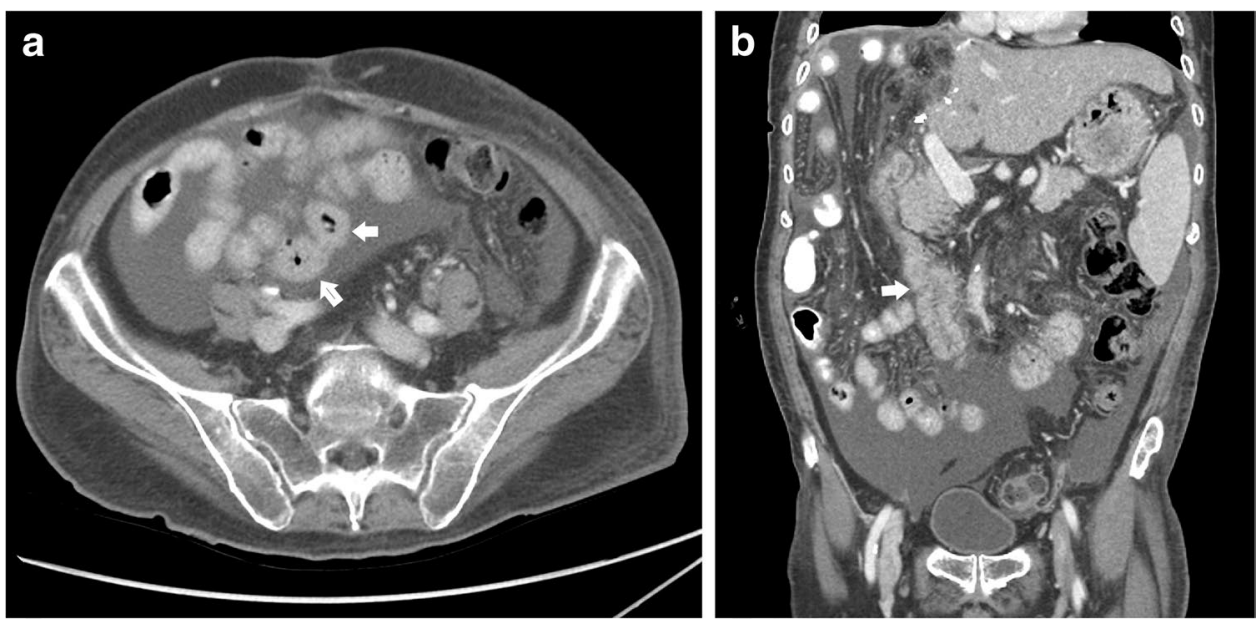
Fig. 5 A 63-year-old male with new wall thickening of the transverse colon (a, arrow). A CT scan performed 4 months prior shows the same segment of transverse colon without wall thickening (b, arrow)
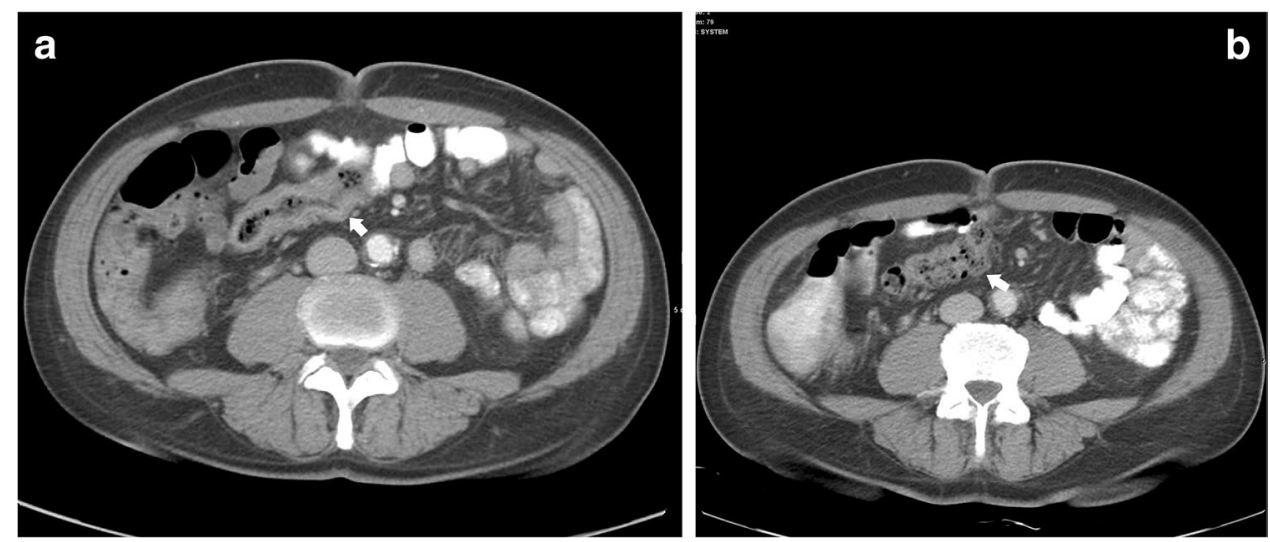

arterial thrombosis [26], and pancreatitis [28]. A study by Shiralkar et al. [5] reported that gastric and bowel abnormalities were seen in $25 \%$ (3/12) of their subjects, while a study by Bhayana et al. found bowel wall abnormalities on $31 \%(13 / 42)$ of their patients who underwent examination with CT. In the latter cohort, some of the patients showed bowel ischemia at surgery, suggesting a possible etiology of the enteritis seen on CT. A separate case report from Chiu et al. [32] reported ischemic enteritis related to COVID-19. Taking these findings into consideration, along with the fact that there is expression of angiotensin-converting enzyme 2 receptors in the GI tract and the presence of detectable SARS-CoV-2 in wastewater $[8,9,33]$, there is reason to believe the virus plays a direct role in the bowel abnormalities seen in our study and in others (Figs. 6, 7, and 8). For practicing emergency radiologists, it is notable that most of our patients were scanned in the outpatient setting (73.0\%) and only a minority had abdominal symptoms (15.9\%), so the imaging findings should not simply be dismissed in asymptomatic patients.

Additional findings such as gallbladder distention without gallbladder thickening, new small volume ascites, and isolated cases of acute pancreatitis and portal vein thrombosis have less clear clinical implications but can be viewed considering the existing literature. Gallbladder distention was established using established measurement threshold criteria, but none of the cases had cholecystitis. In a cohort of 37 patients with abnormal liver function tests (LFTs), Bhayana et al. [6] did report 54\% showing gallbladder distention and biliary sludge on right upper quadrant ultrasound, which the authors of that study took as suggestive of biliary stasis. Although we could not reliably assess for biliary sludge on $\mathrm{CT}$, we did see new gallbladder distention in $7.0 \%$ of patients. The discrepancy in terms of prevalence of this finding between our study and theirs may be that many of our patients were asymptomatic and were scanned as part of routine re-staging rather than for an abnormality of serum LFTs. In addition, gallbladder distention could be affected by fasting status, which could not be accounted for in this retrospective study.

We did also observe one patient with acute pancreatitis in our cohort, a finding that has also been reported in association with COVID-19 infection in rare instances [5, 11]. Our cohort also found a new single case of portal vein thrombosis. Increased prevalence of venous and arterial thrombotic events in the abdomen and pelvis in patients with COVID-19
Fig. 6 A 63-year-old male with bladder cancer and COVID-19 presents with abdominal pain. $\mathrm{CT}$ of the upper abdomen shows enlargement of the pancreatic head with peripancreatic edema and fat infiltration (a, arrow). A comparison scan from 1 month prior shows a normal pancreatic head (b, arrow)
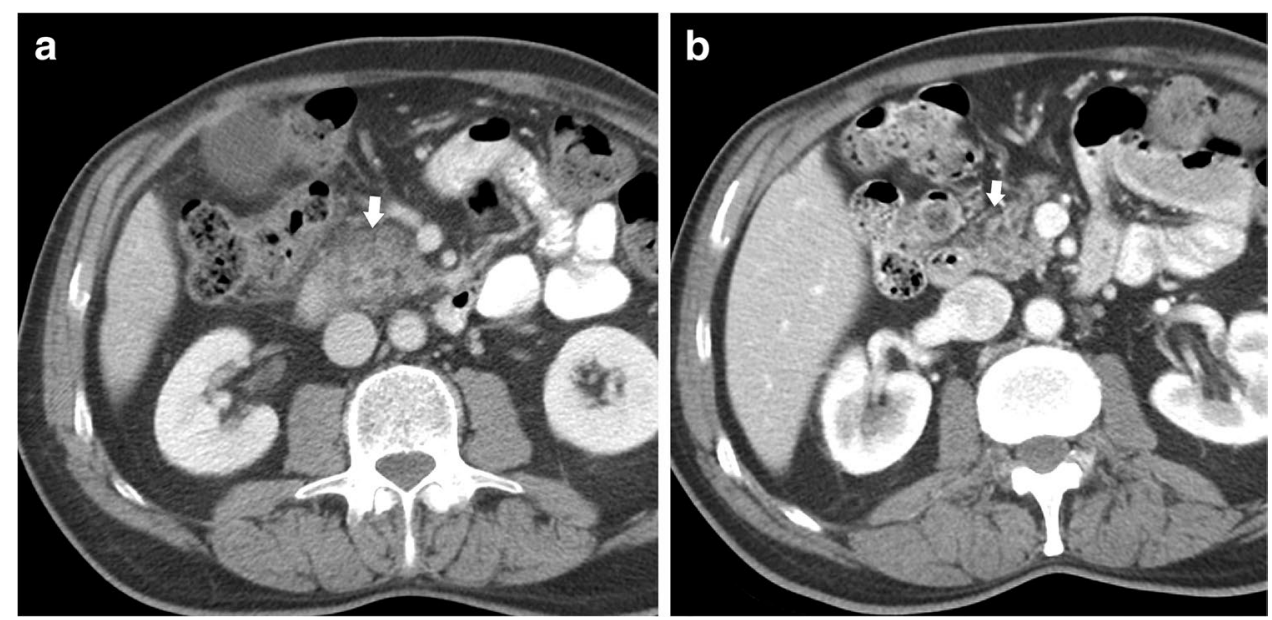
Fig. 7 A 67-year-old female with intrahepatic cholangiocarcinoma and new thrombosis of a branch of the right portal vein (a, arrow). A CT from 2 months prior shows no thrombus (b, arrow)
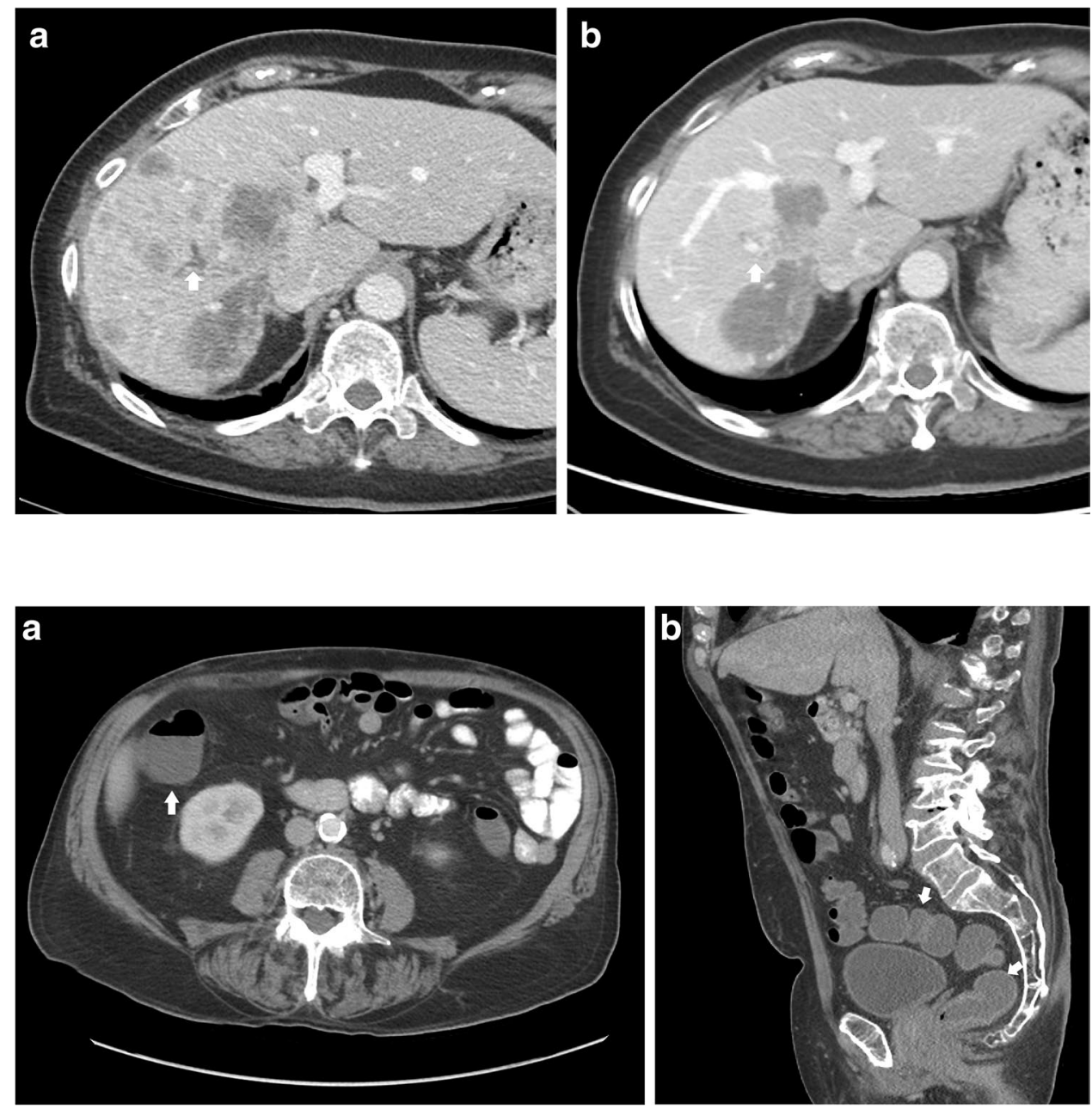

Fig. 8 A 71-year-old male with lung cancer and COVID-19. Axial and sagittal CT images show new fluid-filled ascending (a, arrow) and rectosigmoid (b, arrows) colon has been reported $(34,35)$. However, in our single case, it is difficult to establish a cause and effect relationship between the virus and the portal vein thrombosis, which was seen in the presence of worsening hepatic metastases from intrahepatic cholangiocarcinoma.

Although our study provides added value to the literature, specifically by evaluating new CT findings in a fairly large cohort of patients with SARS-CoV-2 infection, it also has its limitations. First, it is a retrospective study and we are thus reliant on the existing medical records to extract clinical information documented by treating providers. Second, it encompasses patients from a single tertiary academic cancer center that may not be representative of the population of COVID-19 patients at large. Because our patients are all derived from the oncologic population, they may have other reasons (i.e., medications, malignancy) that contribute to imaging abnormalities observed. Third, we lack pathologic correlation for the findings we observe on $\mathrm{CT}$, which limits our ability to draw conclusive cause and effect relationships between the viral infection and imaging abnormalities.

In summary, aside from ground glass opacities in the lung bases, the most common abnormalities seen on abdominopelvic CT in patients with confirmed COVID-19 infection in our cohort involved thickening or fluid-filled hollow viscera, seen in $22.2 \%$. This was slightly less prevalent than in some previously published studies, which may be attributed to the fact that many of our patients were asymptomatic and were scanned as part of their routine cancer care. Less common findings, such as gallbladder distention, new small volume ascites, pancreatitis, and portal vein thrombosis, were also seen in rare instances, but it is not clear whether a causal relationship exists.

\section{Conclusion}

Aside from lung base ground glass opacities, the most common new imaging abnormality on abdominopelvic CT in patients with acute SARS-CoV-2 infection in our cohort was features of gastric or bowel thickening, followed by small volume ascites, gallbladder distention, and isolated cases of pancreatitis and portal vein thrombosis. These new findings overlap with those previously reported that did not have prior imaging for comparison, thus supporting the notion 
that there may be a relationship between SARS-CoV-2 infection and these new abnormal imaging findings. Familiarity with these findings is useful for emergency radiologists, as they may be the first to encounter patients with SARS-CoV-2 infection. Further high-quality investigation into the abdominal manifestations of COVID-19 may be warranted.

Funding This work was supported in part by NCI/NIH Core Grant P30 CA008748.

\section{Declarations}

Conflict of interest The authors declare that they have no conflict of interest.

\section{References}

1. Organization WH (2021) Numbers at a glance. https://www.who. int/emergencies/diseases/novel-coronavirus-2019. Accessed 23 July 2021

2. Bai C, Chotirmall SH, Rello J, Alba GA, Ginns LC, Krishnan JA et al (2020) Updated guidance on the management of COVID-19: from an American thoracic society/European respiratory society coordinated international task force (29 July 2020). Eur Respir Rev. https://doi.org/10.1183/16000617.0287-2020

3. Kapoor MC (2020) Respiratory and cardiovascular effects of COVID-19 infection and their management. J Anaesthesiol Clin Pharmacol 36(Suppl 1):S21-S28

4. Leng L, Cao R, Ma J, Mou D, Zhu Y, Li W et al (2020) Pathological features of COVID-19-associated lung injury: a preliminary proteomics report based on clinical samples. Signal Transduct Target Ther 5(1):240

5. Shiralkar K, Chinapuvvula N, Ocazionez D (2020) Cross-sectional abdominal imaging findings in patients with COVID-19. Cureus 12(8):e9538

6. Bhayana R, Som A, Li MD, Carey DE, Anderson MA, Blake MA et al (2020) Abdominal imaging findings in COVID-19: preliminary observations. Radiology 297(1):E207-E215

7. Safari S, Keyvani H, Alamdari NM, Dehghanian A, Hashemi MR, Honar BN et al (2020) Abdominal surgery in patients with COVID-19: detection of SARS-CoV-2 in abdominal and adipose tissues. Ann Surg. https://doi.org/10.1097/SLA.0000000000 004165

8. Gallardo-Escarate C, Valenzuela-Munoz V, Nunez-Acuna G, Valenzuela-Miranda D, Benaventel BP, Saez-Vera C et al (2020) The wastewater microbiome: a novel insight for COVID-19 surveillance. Sci Total Environ. https://doi.org/10.1016/j.scitotenv. 2020.142867

9. D'Aoust PM, Mercier E, Montpetit D, Jia JJ, Alexandrov I, Neault $\mathrm{N}$ et al (2020) Quantitative analysis of SARS-CoV-2 RNA from wastewater solids in communities with low COVID-19 incidence and prevalence. Water Res 188:116560

10. Zhang H, Kang Z, Gong H, Xu D, Wang J, Li Z, et al. (2020) The digestive system is a potential route of 2019-nCov infection: a bioinformatics analysis based on single-cell transcriptomes. bioRxiv. 2020:2020.01.30.927806.

11. Mazrouei SSA, Saeed GA, Al Helali AA (2020) COVID-19-associated acute pancreatitis: a rare cause of acute abdomen. Radiol Case Rep 15(9):1601-1603
12 Dane B, Smereka P, Wain R, Kim D, Katz DS (2020) Hypercoagulability in patients with coronavirus disease (COVID-19): identification of arterial and venous thromboembolism in the abdomen, pelvis, and lower extremities. Am J Roentgenol. https://doi.org/10.2214/AJR.20.23617

13. Robilotti EV, Babady NE, Mead PA, Rolling T, Perez-Johnston $\mathrm{R}$, Bernardes $\mathrm{M}$ et al (2020) Determinants of COVID-19 disease severity in patients with cancer. Nat Med 26(8):1218-1223

14. Samanta J, Dhar J, Khaliq A, Kochhar R (2020) 2019 Novel coronavirus infection: gastrointestinal manifestations. J Dig Endosc 11(01):13-18

15. Liang W, Feng Z, Rao S, Xiao C, Xue X, Lin Z et al (2020) Diarrhoea may be underestimated: a missing link in 2019 novel coronavirus. Gut 69(6):1141-1143

16. Abolyazid S, Alshareef S, Abdullah N, Khalil A, Hamza N, Salem A (2020) COVID-19 pneumonia identified by CT of the abdomen: a report of three emergency patients presenting with abdominal pain. Radiol Case Rep 15(11):2090-2094

17. Barkmeier DT, Stein EB, Bojicic K, Otemuyiwa B, Vummidi D, Chughtai A et al (2020) Abdominal CT in COVID-19 patients: incidence, indications, and findings. Abdom Radiol (NY). https://doi.org/10.1007/s00261-020-02747-5

18 Funt SA, Cohen SL, Wang JJ, Sanelli PC, Barish MA (2020) Abdominal pelvic CT findings compared between COVID-19 positive and COVID-19 negative patients in the emergency department setting. Abdom Radiol (NY). https://doi.org/10. 1007/s00261-020-02796-w

19. King MJ, Lewis S, El Homsi M, Hernandez Meza G, Bernheim A, Jacobi A et al (2020) Lung base CT findings in COVID-19 adult patients presenting with acute abdominal complaints: case series from a major New York City health system. Eur Radiol 30(12):6685-6693

20. Sendi AA, Saggat DF, Alzahrani SJ (2020) Incidental typical COVID-19 appearance on the lung bases, visualized at abdominal CT for a patient that presented with abdominal pain and nausea. Radiol Case Rep 15(8):1238-1241

21. Siegel A, Chang PJ, Jarou ZJ, Paushter DM, Harmath CB, Arevalo JB et al (2020) Lung base findings of coronavirus disease (COVID-19) on abdominal CT in patients with predominant gastrointestinal symptoms. Am J Roentgenol 215(3):607-609

22. Voutsinas N, Toussie D, Jacobi A, Bernheim A, Chung M (2020) Incidental CT findings in the lungs in COVID-19 patients presenting with abdominal pain. Clin Imaging 67:1-4

23 Gahide G, Frandon J, Vendrell JF (2020) COVID-19 patients presenting with afebrile acute abdominal pain. Clin Med (Lond). https://doi.org/10.7861/clinmed.2020-0150

24. Kecler-Pietrzyk A, Orsi G, Carthy J, Torreggiani WC (2020) Enteritis and severe abdominal pain as the first presentation of COVID-19. Ir Med J 113(6): 102

25 Kim J, Thomsen T, Sell N, Goldsmith AJ (2020) Abdominal and testicular pain: an atypical presentation of COVID-19. Am J Emerg Med 38(7):15421 e1-1542 e3

26 Mahan K, Kabrhel C, Goldsmith AJ (2020) Abdominal pain in a patient with COVID-19 infection: a case of multiple thromboemboli. Am J Emerg Med. https://doi.org/10.1016/j.ajem.2020.05. 054

27. Poggiali E, Ramos PM, Bastoni D, Vercelli A, Magnacavallo A (2020) Abdominal pain: a real challenge in novel COVID-19 infection. Eur J Case Rep Intern Med 7(4):001632

28. Purayil N, Sirajudeen J, Va N, Mathew J (2020) COVID-19 presenting as acute abdominal pain: a case report. Cureus 12(8): $\mathrm{e} 9659$

29. Saeed U, Sellevoll HB, Young VS, Sandbaek G, Glomsaker T, Mala T (2020) COVID-19 may present with acute abdominal pain. Br J Surg 107(7):e186-e187 
30. Walpole SC, McHugh R, Samuel J, Schmid ML (2020) COVID19 presenting as severe, persistent abdominal pain and causing late respiratory compromise in a 33-year-old man. BMJ Case Rep. https://doi.org/10.1136/bcr-2020-236030

31. Zhai LL, Wang W, Wu L, Tang ZG (2020) COVID-19 with abdominal symptoms and acute abdominal pain: a guide to identification for general practice. Br J Gen Pract 70(696):358-359

32 Chiu CY, Sarwal A, Mon AM, Tan YE, Shah V (2020) Gastrointestinal: COVID-19 related ischemic bowel disease. J Gastroenterol Hepatol. https://doi.org/10.1111/jgh.15254

33 de Jong FC, GeurtsvanKessel CH, Molenkamp R, Bangma CH, Zuiverloon TCM (2020) Sewage surveillance system using urological wastewater: key to COVID-19 monitoring? Urol Oncol. https://doi.org/10.1016/j.urolonc.2020.10.008
34. Olson MC, Lubner MG, Menias CO, Mellnick VM, Gettle LM, Kim DH et al (2020) RadioGraphics update: venous thrombosis and hypercoagulability in the abdomen and pelvis-findings in COVID-19. Radiographics 40(5):E24-E28

35. Roncati L, Manenti A, Manco G, Farinetti A (2020) Abdominal aortic thrombosis complicating COVID-19 pneumonia. Ann Vasc Surg 67:8-9

Publisher's Note Springer Nature remains neutral with regard to jurisdictional claims in published maps and institutional affiliations. 